\title{
REPRESENTATION OF SET VALUED OPERATORS
}

\author{
BY
}

\author{
NIKOLAOS S. PAPAGEORGIOU ${ }^{1}$
}

\begin{abstract}
In this paper we prove representation theorems for set valued additive operators acting on the spaces $L_{X}^{1}$ ( $X=$ separable Banach space), $L^{1}$ and $L^{\infty}$. Those results generalize well-known ones for single valued operators and among them the celebrated Dunford-Pettis theorem. The properties of these representing integrals are studied. We also have a differentiability result for multifunctions analogous to the one that says that an absolutely continuous function from a closed interval into a Banach space with the Radon-Nikodym property is almost everywhere differentiable and also it is the primitive of its strong derivative. Finally we have a necessary and sufficient condition for the set of integrable selectors of a multifunction to be $w$-compact in $L_{X}^{1}$. This result is a new very general result about $w$-compactness in the Lebesgue-Bochner space $L_{X}^{1}$.
\end{abstract}

1. Introduction. Integral representation theory has been studied by several authors for linear and nonlinear additive functionals and operators on various function spaces such as Lebesgue-Bochner spaces and Orlicz spaces (see Alò and Korvin [1], Diestel and Uhl [12], Hiai [17] and Mizel and Sundaresan [22]).

In this paper we examine the corresponding problems for additive set valued operator, from which we obtain an integral representation theorem for a lower semicontinuous set valued operator acting on the Lebesgue-Bochner space $L_{X}^{1}(\Omega)$. In fact we show that the representing integrand multifunction has several nice regularity properties. We also extend the celebrated Dunford-Pettis theorem which is a result about the integral representation of a linear continuous operator on $L^{1}$ into a Banach space $X$ (see Diestel and Uhl [12, p. 73]) to the case of an additive set valued operator (also called multioperator). Several versions of this result are presented under a different and very general set of hypotheses.

In $\$ 4$ we examine some additional properties of such representable set valued operators. Also we present a general result about "differentiation" of multifunctions. It is well known that if $X$ is a Banach space with the Radon-Nikodým Property (abbreviated by R.N.P.), then a function $f:[0, T] \rightarrow X$ which is absolutely continuous is almost everywhere strongly differentiable, $\dot{f}(\cdot)$ is Bochner integrable and furthermore we can write that $f(t)=f(0)+\int_{0}^{t} \dot{f}(\tau) d \tau$. This result was first proved by Gelfand [21] for the case $X$ is reflexive and later it was realized that the result is

Received by the editors October 21, 1984.

1980 Mathematics Subject Classification. Primary 28A45, 46G10, 46E30.

Key words and phrases. Set valued operator, lifting, support function, measurable multifunction integrable selector, Radon-Nikodým property, weak compactness, multimeasure.

${ }^{1}$ Research supported by N.S.F. Grant D.M.S.-8403135. 
still true under the more general hypothesis that $X$ has the R.N.P. In fact it turns out that this is also a sufficient condition for a space to be a Radon-Nikodým space. A multivalued version of this result was obtained by Artstein [2] for $X=\mathbf{R}^{n}$ and by Castaing [5] for $X$ being a separable dual Banach space and under quite restrictive additional hypotheses. Here we relax all those assumptions and we present a more general differentiability result. Finally we also have an interesting result about the set of integrable selectors of a measurable multifunction.

In the next section we establish our terminology and notations and we present some auxiliary material that we will need in the sequel.

2. Preliminaries. Let $(\Omega, \Sigma, \mu)$ be a complete, finite measure space and $X$ a separable Banach space. Additional hypotheses will be introduced as needed. By $X^{*}$ we will denote the topological dual of $X$. We will use the following notation.

$P_{f(c)}(X)=\{A \subseteq X$ : nonempty, closed, (convex) $\}$.

$P_{k(c)}(X)=\{A \subseteq X$ : nonempty, compact, (convex) $\}$.

A $\mathrm{w}$ in front of $f$ (resp. $k$ ) will mean that the closedness (resp. compactness) is with respect to the weak topology w $\left(X, X^{*}\right)$. If $A \in P_{f}(X)$, then we write $|A|=$ $\sup _{x \in A}\|x\|$. Also $\sigma_{A}\left(x^{*}\right)=\sup _{x \in A}\left(x^{*}, x\right)$ is the support function of $A$.

A multifunction $F: \Omega \rightarrow P_{f}(X)$ is said to be measurable if it satisfies any of the following three equivalent conditions:

(1) $F^{-}(U)=\{\omega \in \Omega: F(\omega) \cap U \neq \varnothing\} \in \Sigma$ for all $U \subseteq X$ open.

(2) $\omega \rightarrow d(x, F(\omega))$ is $\Sigma$-measurable for all $x \in X$.

(3) There exists a sequence $\left\{f_{n}(\cdot)\right\}_{n \geqslant 1}$ of measurable functions $f_{n}: \Omega \rightarrow X$ s.t. $f_{n}(\omega) \in F(\omega)$ and $F(\omega)=\operatorname{cl}\left\{f_{n}(\omega)\right\}_{n \geqslant 1}$ for all $\omega \in \Omega$ (Castaing representation).

A measurable function $f: \Omega \rightarrow X$ s.t. $f(\omega) \in F(\omega)$ for all $\omega \in \Omega$ is said to be a selector of $F(\cdot)$. By $S_{F}^{1}$ we denote all selectors of $F(\cdot)$ belonging to $L_{X}^{1}(\Omega)$, i.e., $S_{F}^{1}=\left\{f(\cdot) \in L_{X}^{1}(\Omega): f(\omega) \in F(\omega) \mu\right.$-a.e. $\}$. We will say that $F(\cdot)$ is integrably bounded if and only if $|F(\cdot)| \in L^{1}(\Omega)$. In this case the Kuratowski-Ryll-Nardzewski selection theorem tells us that $S_{F}^{1} \neq \varnothing$. Using $S_{F}^{1}$ we can define an integral for multifunctions. This was first introduced by Aumann [3] for the case $X=\mathbf{R}^{n}$ and is the natural extension of the single valued integral and of the Minkowski sum of sets. So we have

$$
\int_{\Omega} F(\omega) d \mu(\omega)=\left\{\int_{\Omega} f(\omega) d \mu(\omega): f \in S_{F}^{1}\right\},
$$

where the vector integrals are taken in the sense of Bochner. This integral turned out to be the appropriate analytical tool in several applied fields like optimization, optimal control, mathematical economics and statistics. For more details about measurable multifunctions and their integrals we refer to Castaing and Valadier [6], Hiai and Umegaki [18], Himmelberg [19] and Rockafellar [25].

Finally we would like to introduce a notion of convergence of nonempty, closed sets in $X$ which we will call Kuratowski-Mosco convergence (K-M convergence) and which is more general then convergence in the Hausdorff metric. Let $\left\{A_{n}\right\}_{n \geqslant 1}$ be a sequence in $P_{f}(X)$. Let

$$
\mathrm{s}-\liminf _{n \rightarrow \infty} A_{n}=\left\{x \in X: x=\operatorname{s}_{n \rightarrow \infty} \lim _{n}, x_{n} \in A_{n}, n \geqslant 1\right\}
$$


(here s denotes the strong (norm) topology on $X$ ) and

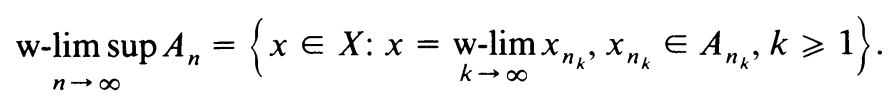

We will say that $A_{n}$ converges to $A \in P_{f}(X)$ in the Kuratowski-Mosco sense (written as $A_{n} \stackrel{\mathrm{K}-\mathrm{M}}{\rightarrow} A$ as $\left.n \rightarrow \infty\right)$ if and only if w-lim $\sup _{n \rightarrow \infty} A_{n}=A=\mathrm{s}-\lim \inf _{n \rightarrow \infty} A_{n}$. For more details we refer to Mosco [23].

3. Representation theorems. Consider a set valued operator $T: L_{X}^{1}(\Omega) \rightarrow 2^{X} \backslash\{\varnothing\}$. We will say that $T(\cdot)$ is additive if $T\left(f_{1}+f_{2}\right)=T\left(f_{1}\right)+T\left(f_{2}\right)$ for all $f_{1}(\cdot)$, $f_{2}(\cdot) \in L_{X}^{1}(\Omega)$ s.t. $\operatorname{supp}\left(f_{1}\right) \cap \operatorname{supp}\left(f_{2}\right)=\varnothing$ (i.e. $f_{1}, f_{2}$ have disjoint supports). We will say that $T(\cdot)$ is sublinear if it is additive in the above sense and positively homogeneous.

We start with a lemma that is also interesting in its own sake.

LeMma. If $F: \Omega \times X \rightarrow P_{\mathrm{w} k c}(X)$ is a multifunction s.t.

(1) $F(\cdot, x)$ is measurable for all $x \in X$,

(2) $x \rightarrow \sigma_{F(\omega, x)}\left(x^{*}\right)$ is l.s.c. for all $\omega \in \Omega$ and all $x^{*} \in X^{*}, \sigma_{F(\cdot, \cdot)}\left(x^{*}\right)$ separable then for all $f: \Omega \rightarrow X$ measurable, $\omega \rightarrow F(\omega, f(\omega))$ is measurable.

Proof. Consider the function $\varphi_{x^{*}}(\omega, x)=\sigma_{F(\omega, x)}\left(x^{*}\right)$ and fix $x^{*} \in X^{*}$. We claim that $\varphi_{x^{*}}(\cdot, \cdot)$ is a normal integrand. From Rockafellar [25] we know that it suffices to show that the multifunction $\omega \rightarrow R(\omega)=$ epi $\varphi_{x^{*}}(\omega, \cdot)$ is closed valued and measurable. From hypothesis (2) we have that $x \rightarrow \varphi_{x^{*}}(\omega, x)$ is l.s.c. and so $R(\cdot)$ is closed valued. Next note that $\varphi_{x^{*}}(\omega, \cdot)$ is finite valued for all $\omega \in \Omega$. So $\operatorname{dom} R=\Omega$. Let $D=\left\{\left(x_{n}, \lambda_{n}\right) \in X \times \mathbf{R} ; n \geqslant 1\right\}$ be a countable dense set in $X \times \mathbf{R}$ and define the functions $g_{n}(\omega)=\left(x_{n}, \max \left(\varphi_{x^{*}}\left(\omega, x_{n}\right), \lambda_{n}\right)\right)$. Clearly $g_{n}(\cdot)$ is a Castaing representation for $R(\cdot)$. So $\omega \rightarrow R(\omega)$ is measurable. Thus we deduce that $\varphi_{x^{*}}(\cdot, \cdot)$ is a normal integrand which means that it is $(\Sigma \times B(X))$-measurable. So $\omega \rightarrow$ $\varphi_{x^{*}}(\omega, f(\omega))=\sigma_{F(\omega, f(\omega))}\left(x^{*}\right)$ is measurable and this means, by Theorem III-37 of Castaing and Valadier [6], that $\omega \rightarrow F(\omega, f(\omega))$ is measurable. Q.E.D.

REMARK. In the above proof we also got that $(\omega, x) \rightarrow F(\omega, x)$ is $(\Sigma \times B(X))$ measurable. Also if $F(\omega, \cdot)$ is 1.s.c. (in the set valued sense, see Castaing and Valadier [6]), then $x \rightarrow \sigma_{F(\omega, x)}\left(x^{*}\right)$ is l.s.c.

Now we are ready for the first representation theorem.

TheOREM 3.1. Assume that $X$ is reflexive. If $T: L_{X}^{1}(\Omega) \rightarrow P_{f c}(X)$ is sublinear and l.s.c., and $T(f) \subseteq \int_{\Omega}\|f(\omega)\| W(\omega) d \mu(\omega)$ for all $f(\cdot) \in L_{X}^{1}(\Omega)$, where $W: \Omega \rightarrow$ $P_{\mathrm{w} k c}(X)$ is integrably bounded, then there exists $F: \Omega \rightarrow P_{\mathrm{w} k c}(X)$ s.t.

(a) for all $x \in X, F(\cdot, \cdot)$ is integrably bounded by $p(\cdot) \in L^{1}(\Omega)$,

(b) for all $\omega \in \Omega, F(\omega, \cdot)$ is l.s.c. from $X$ into $(X, \mathrm{w})$ and $F(\omega, 0)=\{0\} \mu$-a.e.,

(c) for all $f(\cdot) \in L_{X}^{1}(\Omega)$ we have that $F(\omega, f(\omega)) \subseteq\|f(\omega)\| W(\omega) \mu$-a.e. and $T(f)=\int_{\Omega} F(\omega, f(\omega)) d \mu(\omega)$.

Proof. In the light of the previous lemma and of the Kuratowski-Ryll-Nardzewski selection theorem, the integral in the conclusion of the theorem is nonempty. 
For every $x^{*} \in X^{*}$ consider the function $f \rightarrow \sigma_{T(f)}\left(x^{*}\right)$. Clearly this is sublinear since $T(\cdot)$ is. We claim that it is also lower semicontinuous (1.s.c.). To show this we work as follows. Let $f_{n} \stackrel{L_{x}^{1}}{\rightarrow} f$ and let $x \in \mathrm{s}-\lim _{n \rightarrow \infty} T\left(f_{n}\right)$. Then we can find $x_{n} \in T\left(f_{n}\right)$ s.t. $x_{n} \stackrel{\mathrm{s}}{\rightarrow} x \Rightarrow\left(x^{*}, x_{n}\right) \rightarrow\left(x^{*}, x\right)$. But $\left(x^{*}, x_{n}\right) \leqslant \sigma_{T\left(f_{n}\right)}\left(x^{*}\right)$ for all $n \geqslant 1 \Rightarrow\left(x^{*}, x\right)$ $\leqslant \lim _{n \rightarrow \infty} \sigma_{T\left(f_{n}\right)}\left(x^{*}\right)$. Since $x \in \mathrm{s}-\lim _{n \rightarrow \infty} T\left(f_{n}\right)$ was arbitrary we conclude that $\sigma_{\mathrm{s}-\lim _{n \rightarrow \infty} T\left(f_{n}\right)}\left(x^{*}\right) \leqslant \lim _{n \rightarrow \infty} \sigma_{T\left(f_{n}\right)}\left(x^{*}\right)$. On the other hand, since by hypothesis $T(\cdot)$

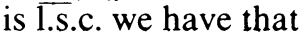

$$
T(f) \subseteq \mathrm{s}-\lim _{n \rightarrow \infty} T\left(f_{n}\right) \Rightarrow \sigma_{T(f)}\left(x^{*}\right) \leqslant \sigma_{s-\lim _{n \rightarrow \infty} T\left(f_{n}\right)}\left(x^{*}\right) .
$$

So indeed $f \rightarrow \sigma_{T(f)}\left(x^{*}\right)$ is 1.s.c. Then Theorem 5.1 of Hiai [17] tells us that for all $x^{*} \in X^{*}$ there exists $\varphi_{x^{*}}: \Omega \times \Sigma \rightarrow \overline{\mathbf{R}}$ normal s.t. $\varphi_{x^{*}}(\omega, 0)=0 \mu$-a.e. and $\sigma_{T(f)}\left(x^{*}\right)$ $=\int_{\Omega} \varphi_{x^{*}}(\omega, x) d \mu(\omega)$. Fix $x^{*} \in X^{*}$ and $x \in X$. Then by letting $f=\chi_{A} x$, where $A \in \Sigma$, we have that

$$
\sigma_{T\left(\chi_{A} x\right)}\left(x^{*}\right)=\int_{A} \varphi_{x^{*}}(\omega, x) d \mu(\omega) \leqslant\|x\|\left\|x^{*}\right\| \int_{A}|W(\omega)| d \mu(\omega) .
$$

Since this is true for all $A \in \Sigma$ we have that $\varphi_{x^{*}}(\omega, x) \leqslant\|x\|\left\|x^{*}\right\||W(\omega)|$ for all $\omega \in \Omega \backslash N_{x x^{*}}$ with $\mu\left(N_{x x^{*}}\right)=0$. Set $\Omega_{n}=\{\omega \in \Omega: n \leqslant|W(\omega)|<n+1\} ; \varphi_{x^{*}}(\cdot, x)$ is essentially bounded on every $\Omega_{n}$. Let $\rho$ be a positive, linear lift of $L^{\infty}\left(\Omega_{n}, \Sigma_{n},\left.\mu\right|_{\Omega_{n}}\right)$ (where $\Sigma_{n}=\Sigma \cap \Omega_{n}$ ). Such a lift exists by Ionescu-Tulcea [20]. Let $\hat{\varphi}_{x^{*}}(\omega, x)=$ $\rho\left[\varphi_{x^{*}}(\omega, x)\right]$. Define a function on all $\Omega$ by piecing those lifts together. Then since

$$
\int_{A} \varphi_{x_{1}^{*}+x_{2}^{*}}(\omega, x) d \mu(\omega) \leqslant \int_{A}\left[\varphi_{x_{1}^{*}}(\omega, x)+\varphi_{x_{2}^{*}}(\omega, x)\right] d \mu(\omega)
$$

and

$$
\int_{A} \varphi_{\lambda x^{*}}(\omega, x) d \mu(\omega)=\lambda \int_{A} \varphi_{x^{*}}(\omega, x) d \mu(\omega)
$$

for all $A \in \Sigma$, we get that $\hat{\varphi}_{x_{1}^{*}+x_{2}^{*}}(\omega, x) \leqslant \hat{\varphi}_{x_{1}^{*}}(\omega, x)+\hat{\varphi}_{x_{2}^{*}}(\omega, x)$ and $\hat{\varphi}_{\lambda x^{*}}(\omega, x)=$ $\lambda \hat{\varphi}_{x^{*}}(\omega, x)$ for all $\omega \in \Omega$. Thus $x^{*} \rightarrow \hat{\varphi}_{x^{*}}(\omega, x)$ is a sublinear function. In addition it is bounded and so it is continuous. Let

$$
F(\omega, x)=\left\{z \in X:\left(x^{*}, z\right) \leqslant \hat{\varphi}_{x^{*}}(\omega, x) \text { for all } x^{*} \in X^{*}\right\} .
$$

Using the continuity of $\hat{\varphi}_{1}(\omega, x)$ we can write that

$$
F(\omega, x)=\bigcap_{n \geqslant 1}\left\{z \in X:\left(x_{n}^{*}, Z\right) \leqslant \hat{\varphi}_{x_{n}^{*}}(\omega, x)\right\},
$$

where $\left\{x_{n}^{*}\right\}_{n \geqslant 1}$ is dense in $X^{*}$. So $(\omega, x) \rightarrow F(\omega, x)$ is measurable with values in $P_{f c}(X)$. Then we have

$$
\begin{aligned}
\sigma_{T(f)}\left(x^{*}\right) & =\int_{\Omega} \varphi_{x^{*}}(\omega, f(\omega)) d \mu(\omega)=\int_{\Omega} \sigma_{F(\omega, f(\omega))}\left(x^{*}\right) d \mu(\omega) \\
& =\sigma_{f_{\Omega} F(\omega, f(\omega))}\left(x^{*}\right) \Rightarrow T(f)=\operatorname{cl} \int_{\Omega} F(\omega, f(\omega)) d \mu(\omega) .
\end{aligned}
$$


Also note that

$$
\begin{aligned}
\int_{\Omega} F(\omega, f(\omega)) d \mu(\omega) & \subseteq \int_{\Omega}\|f(\omega)\| W(\omega) d \mu(\omega) \Rightarrow \int_{\Omega} \sigma_{F(\omega, f(\omega))}\left(x^{*}\right) d \mu(\omega) \\
& \leqslant \int_{\Omega}\|f(\omega)\| \sigma_{W(\omega)}\left(x^{*}\right) d \mu(\omega) .
\end{aligned}
$$

Since this is true for all $f \in L_{X}^{1}(\Omega)$, from Lemma 4.2 of Hiai [17] we deduce that $\sigma_{F(\omega, f(\omega))}\left(x^{*}\right) \leqslant\|f(\omega)\| \sigma_{W(\omega)}\left(x^{*}\right)$ for all $\omega \in \Omega \backslash N_{x^{*}}$ with $\mu\left(N_{x^{*}}\right)=0$. For the dense set $\left\{x_{n}^{*}\right\}_{n \geqslant 1}$ clearly we can say that $\sigma_{F(\omega, f(\omega))}\left(x_{n}^{*}\right) \leqslant\|f(\omega)\| \sigma_{W(\omega)}\left(x_{n}^{*}\right) \mu$-a.e. for all $n \geqslant 1$. Then from the continuity of the support functions we deduce that $\sigma_{F(\omega, f(\omega))}\left(x^{*}\right) \leqslant\|f(\omega)\| \sigma_{W(\omega)}\left(x^{*}\right) \mu$-a.e. for all $x^{*} \in X^{*}$. Since both sets are closed, convex we conclude that $F(\omega, f(\omega)) \subseteq\|f(\omega)\| W(\omega) \mu$-a.e. So $F(\omega, f(\omega))$ has values in $P_{\mathrm{w} k c}(X)$ for $\mu$-almost all $\omega \in \Omega$. Hence using the lemma and Theorem 4.2 of this paper we get $\int_{\Omega} F(\omega, f(\omega)) d \mu(\omega) \in P_{\mathrm{w} k c}(X)$ and so $T(f)=\int_{\Omega} F(\omega, f(\omega)) d \mu(\omega)$.

Finally we have to show that $F(\omega, \cdot)$ is 1 .s.c. from $X$ into $(X, \mathrm{w})=X$ with the weak topology, and that $F(\omega, 0)=\{0\} \mu$-a.e. The second conclusion is immediate because we have that

$$
\sigma_{F(\omega, 0)}\left(x^{*}\right)=\varphi_{x^{*}}(\omega, 0)=0 \quad \mu \text {-a.e. }
$$

Since this is true for all $x^{*} \in X^{*}$ we deduce that $F(\omega, 0)=\{0\} \mu$-a.e.

Next note that for $x \in B_{1}=$ unit ball of $X$ we have that $F(\omega, x) \subseteq W(\omega)$. Also we know that $x \rightarrow \sigma_{F(\omega, x)}\left(x^{*}\right)$ is 1.s.c. for all $\omega \in \Omega$ and all $x^{*} \in X^{*}$. So we can invoke Theorem II-21 of Castaing and Valadier [6] and deduce that, outside a $\mu$-null set $N, F(\omega, \cdot)$ is $1 . s . c$. from $B_{1}$ into $(X, \mathrm{w})=$ the space $X$ with the weak topology. By modifying $F(\cdot, x)$ appropriately on $N$ (for example, set $F(\omega, x)=W(\omega)$ for all $\omega \in N)$ we can say that $F(\omega, \cdot)$ is 1 .s.c. from $B_{1}$ to $(X, w)$ for all $\omega \in \Omega$. We will show that $F(\omega, \cdot)$ is l.s.c. on all of $X$. Note that $F(\omega, \cdot)$ is positively homogeneous since $T(\cdot)$ is. So if $x_{n} \stackrel{s}{\rightarrow} x$ and recalling that the weak topology on $W(\omega)$ is metrizable we have that

$$
\begin{aligned}
\mathrm{w}-\underset{n \rightarrow \infty}{\lim } F\left(\omega, x_{n}\right) & =\|x\| \frac{1}{\|x\|} \underset{n \rightarrow \infty}{\mathrm{w}-}-\frac{\lim }{n \rightarrow \infty}\left(\omega, x_{n}\right)=\|x\| \underset{n \rightarrow \infty}{-\frac{\lim }{\| x}} \frac{1}{\|x\|} F\left(\omega, x_{n}\right) \\
& =\|x\| \underset{n \rightarrow \infty}{-\frac{\lim }{\rightarrow \infty}} F\left(\omega, \frac{x_{n}}{\left\|x_{n}\right\|}\right) .
\end{aligned}
$$

Since $x_{n} /\left\|x_{n}\right\| \rightarrow x /\|x\|$ and $F(\omega, \cdot)$ is l.s.c. from $B_{1}$ to $X_{\mathrm{w}}$ we have

$$
\mathrm{w}-\underset{n \rightarrow \infty}{\lim } F\left(\omega, \frac{x_{n}}{\left\|x_{n}\right\|}\right) \supseteq F\left(\omega, \frac{x}{\|x\|}\right)=\frac{1}{\|x\|} F(\omega, x) .
$$

Thus we get that

$$
\mathrm{w}-\lim _{n \rightarrow \infty} F\left(\omega, x_{n}\right) \supseteq\|x\| \frac{1}{\|x\|} F(\omega, x)=F(\omega, x)
$$

which by Delahaye and Denel [10] tells us that $F(\omega, \cdot)$ is 1.s.c. from $X$ into $X_{\mathrm{w}}$ for all $\omega \in \Omega$. Q.E.D. 
Next we will prove the announced multivalued generalization of the Dunford-Pettis theorem. Following Costé and Pallu de la Barrière [8] we will denote by $H\left(X, X^{*}\right)$ the closed, convex, weakly locally compact, nonempty subsets of $X$, which contain no straight line. Our result also generalizes significantly Theorem 1 of Castaing [4].

So we have

Theorem 3.2. If $T: L^{1}(\Omega) \rightarrow P_{f c}(X)$ is linear (i.e. additive and homogeneous), $T(f) \subset\|f\|_{1} Q$, where $Q$ is in $H\left(X, X^{*}\right)$ and is also bounded, and $f \rightarrow \sigma_{T(f)}\left(x^{*}\right)$ is continuous for all $x^{*} \in X^{*}$, then there exists $F: \Omega \rightarrow H\left(X, X^{*}\right)$ scalarly integrable s.t. $T(f)=\operatorname{cl} \int_{\Omega} f(\omega) F(\omega) d \mu(\omega)$ for all $f(\cdot) \in L^{1}(\Omega)$.

Proof. Consider the map $A \rightarrow T\left(\chi_{A}\right)$. We claim that this is a weak set valued measure in the sense of Costé and Pallu de la Barrière [8]. For that purpose let $\left\{A_{n}\right\}_{n \geqslant 1}$ be a sequence of mutually disjoint $\Sigma$-sets, and let $A=\cup_{n=1}^{\infty} A_{n}$. Let $S_{N}=\bigcup_{n=1}^{N} A_{n}$. Then $T\left(\chi_{A}\right)=T\left(\chi_{S_{N}}+\chi_{S_{N}^{c}}\right)=T\left(\chi_{S_{N}}\right)+T\left(\chi_{S_{N}^{c}}\right)$. Note that $T\left(\chi_{S_{N}}\right)$ $=\sum_{n=1}^{N} T\left(\chi_{A_{n}}\right)$. So we have

$$
T\left(\chi_{A}\right)=\sum_{n=1}^{N} T\left(\chi_{A_{n}}\right)+T\left(\chi_{S_{N}^{c}}\right) \Rightarrow \sigma_{T\left(\chi_{A}\right)}=\sum_{n=1}^{N} \sigma_{T\left(\chi_{A_{n}}\right)}+\sigma_{T\left(\chi_{\left.S_{N}^{c}\right)}\right.} .
$$

But $\chi_{S_{N}^{*}} \stackrel{L^{1}(\Omega)}{\rightarrow} 0$ as $N \rightarrow \infty$ and so $\sigma_{T\left(\chi_{\left.S_{N}\right)}\right)}\left(x^{*}\right) \rightarrow 0$ as $N \rightarrow \infty$ for all $x^{*} \in X^{*}$. Also $\chi_{S_{N}} \stackrel{L^{1}(\Omega)}{\rightarrow} \chi_{A}$. So for all $x^{*} \in X^{*}$

$$
\sigma_{T\left(\chi_{S_{N}}\right)}\left(x^{*}\right)=\sum_{n=1}^{N} \boldsymbol{\sigma}_{T\left(\chi_{A_{n}}\right)}\left(x^{*}\right) \rightarrow \sigma_{T\left(\chi_{A}\right)}\left(x^{*}\right) \quad \text { as } N \rightarrow \infty .
$$

Therefore

$$
\sigma_{T\left(\chi_{A}\right)}\left(x^{*}\right)=\lim _{N \rightarrow \infty} \sum_{n=1}^{N} \sigma_{T\left(\chi_{A_{n}}\right)}\left(x^{*}\right)=\sum_{n=1}^{\infty} \sigma_{T\left(\chi_{A_{n}}\right)}\left(x^{*}\right) .
$$

Furthermore $T(\chi$.$) is \mu$-continuous, because if $\mu(A)=0$ we have that $T\left(\chi_{A}\right) \subseteq$ $\left\|\chi_{A}\right\|_{1} Q=0 . Q=\{0\}$. So we can apply Theorem 3.1 of Costé and Pallu de la Barrière [8] and get that there exists $F: \Omega \rightarrow H\left(X, X^{*}\right)$ scalarly measurable (and so measurable by Theorem III-37 of Castaing and Valadier [6]) and scalarly quasintegrable and for which we have

$$
\sigma_{T\left(\chi_{A}\right)}\left(x^{*}\right)=\int_{A} \sigma_{F(\omega)}\left(x^{*}\right) d \mu(\omega)=\sigma_{\Omega_{\Omega} \chi_{A}(\omega) F(\omega) d \mu(\omega)}\left(x^{*}\right)
$$

for all $x^{*} \in X^{*}$. In fact, $\sigma_{F(\cdot)}\left(x^{*}\right)$ is integrable since $Q$ is bounded, i.e. $F(\cdot)$ is scalarly integrable. So we have

$$
T\left(\chi_{A}\right)=\operatorname{cl} \int_{\Omega} \chi_{A}(\omega) F(\omega) d \mu(\omega) .
$$

Now let $s(\cdot)=\sum_{k=1}^{N} \chi_{A_{k}}(\cdot) r_{k}$ be a nonnegative simple function. Then

$$
\begin{aligned}
T(s) & =\sum_{k=1}^{N} T\left(\chi_{A_{k}} r_{k}\right)=\sum_{k=1}^{N} \mathrm{cl} \int_{A_{k}} r_{k} F(\omega) d \mu(\omega) \\
& =\operatorname{cl} \sum_{k=1}^{N} \int_{A_{k}} r_{k} F(\omega) d \mu(\omega)=\operatorname{cl} \int_{\Omega} s_{n}(\omega) F(\omega) d \mu(\omega) .
\end{aligned}
$$


For an arbitrary simple function $s(\cdot)$ write $s=s^{+}-s^{-}$. Because of the additivity of $T(\cdot)$ we have that

$$
\begin{aligned}
T(s) & =T\left(s^{+}-s^{-}\right)=T\left(s^{+}\right)-T\left(s^{-}\right) \\
& =\operatorname{cl} \int_{\Omega} s^{+}(\omega) F(\omega) d \mu(\omega)-\operatorname{cl} \int_{\Omega} s^{-}(\omega) F(\omega) d \mu(\omega) \\
& =\operatorname{cl}\left[\int_{\Omega} s^{+}(\omega) F(\omega) d \mu(\omega)-\int_{\Omega} s^{-}(\omega) F(\omega) d \mu(\omega)\right] .
\end{aligned}
$$

Next let $f(\cdot) \in L^{1}(\Omega)$ and let $\left\{s_{n}(\cdot)\right\}_{n \geqslant 1}$ be simple functions s.t.

$$
s_{n} \stackrel{L^{1}(\Omega)}{\rightarrow} f, \quad s_{n}^{+} \stackrel{L^{1}(\Omega)}{\rightarrow} f^{+} \text {and } s_{n}^{-} \stackrel{L^{1}(\Omega)}{\rightarrow} f^{-}
$$

and also converge pointwise. Then $\sigma_{T\left(s_{n}\right)}\left(x^{*}\right) \rightarrow \sigma_{T(f)}\left(x^{*}\right)$ for all $x^{*} \in X^{*}$. Note that

$$
\begin{aligned}
\sigma_{T\left(s_{n}\right)}\left(x^{*}\right) & =\sigma_{T\left(s_{n}^{+}-s_{n}^{-}\right)}\left(x^{*}\right)=\sigma_{T\left(s_{n}^{+}\right)-T\left(s_{n}^{-}\right)}\left(x^{*}\right) \\
& =\sigma_{T\left(s_{n}^{+}\right)}\left(x^{*}\right)+\sigma_{-T\left(s_{n}^{-}\right)}\left(x^{*}\right)=\sigma_{T\left(s_{n}^{+}\right)}\left(x^{*}\right)+\sigma_{T\left(s_{n}^{-}\right)}\left(-x^{*}\right) .
\end{aligned}
$$

But we already saw that

$$
\sigma_{T\left(s_{n}^{+}\right)}\left(x^{*}\right)=\sigma_{\mathrm{cl} \int_{\Omega} s_{n}^{+} F}\left(x^{*}\right) \text { and } \sigma_{T\left(s_{n}^{-}\right)}\left(x^{*}\right)=\sigma_{\mathrm{cl} \int_{\Omega} s_{n}^{-} F}\left(-x^{*}\right) .
$$

At this point observe that

$$
\begin{aligned}
\left|\sigma_{\mathrm{cl} \int_{\Omega} s_{n}^{+} F}\left(x^{*}\right)-\sigma_{\mathrm{cl} \int_{\Omega} f^{+} F}\left(x^{*}\right)\right| & =\left|\int_{\Omega}\left(\sigma_{s_{n}^{+}(\omega) F(\omega)}\left(x^{*}\right)-\sigma_{f^{+}(\omega) F(\omega)}\left(x^{*}\right)\right) d \mu(\omega)\right| \\
& =\left|\int_{\Omega}\left(s_{n}^{+}(\omega) \sigma_{F(\omega)}\left(x^{*}\right)-f^{+}(\omega) \sigma_{F(\omega)}\left(x^{*}\right)\right) d \mu(\omega)\right| \\
& \leqslant \int_{\Omega}\left|s_{n}^{+}(\omega)-f^{+}(\omega)\right|\left|\sigma_{F(\omega)}\left(x^{*}\right)\right| d \mu(\omega) .
\end{aligned}
$$

Invoking the Lebesgue dominated convergence theorem we get that

$$
\begin{gathered}
\lim _{n \rightarrow \infty}\left|\int_{\Omega}\left(s_{n}^{+}(\omega) \sigma_{F(\omega)}\left(x^{*}\right)-f^{+}(\omega) \sigma_{F(\omega)}\left(x^{*}\right)\right) d \mu(\omega)\right| \\
=0 \Rightarrow \sigma_{\mathrm{cl} \int_{\Omega} s_{n}^{+} F}\left(x^{*}\right) \rightarrow \sigma_{\mathrm{cl} \int_{\Omega} f^{+} F}\left(x^{*}\right) \text { as } n \rightarrow \infty .
\end{gathered}
$$

Similarly we can get that

$$
\sigma_{\mathrm{cl} \int_{\Omega} s_{n}^{-} F}\left(x^{*}\right) \rightarrow \sigma_{\mathrm{cl} \int_{\Omega} f^{-} F}\left(x^{*}\right) \text { as } n \rightarrow \infty
$$

for all $x^{*} \in X^{*}$. So

$$
\begin{aligned}
\sigma_{T\left(s_{n}\right)}\left(x^{*}\right) & =\sigma_{T\left(s_{n}^{+}\right)}\left(x^{*}\right)+\sigma_{T\left(s_{n}^{-}\right)}\left(-x^{*}\right) \\
& =\sigma_{\mathrm{cl} \int_{\Omega} s_{n}^{+} F}\left(x^{*}\right)+\sigma_{\mathrm{cl} \int_{\Omega} s_{n}^{-} F}\left(-x^{*}\right) \rightarrow \sigma_{\mathrm{cl} \int_{\Omega} f^{+} F}\left(x^{*}\right)+\sigma_{\mathrm{cl} \int_{\Omega} f^{-} F}\left(-x^{*}\right) \\
& =\sigma_{\mathrm{cl} \int_{\Omega} f^{+} F}\left(x^{*}\right)+\sigma_{-\mathrm{cl} \int_{\Omega} f^{-} F}\left(x^{*}\right)=\int_{\Omega} \sigma_{f^{+}(\omega) F(\omega)-f^{-}(\omega) F(\omega)}\left(x^{*}\right) d \mu(\omega) \\
& =\int_{\Omega} \sigma_{f(\omega) F(\omega)}\left(x^{*}\right) d \mu(\omega)=\sigma_{\mathrm{cl} \int_{\Omega} f F}\left(x^{*}\right) .
\end{aligned}
$$

On the other hand, we know that $\sigma_{T\left(s_{n}\right)}\left(x^{*}\right) \rightarrow \sigma_{T(f)}\left(x^{*}\right)$ as $n \rightarrow \infty$. Thus $\sigma_{T(f)}\left(x^{*}\right)$ $=\sigma_{\mathrm{cl} \int_{\Omega} f}\left(x^{*}\right)$ for all $x^{*} \in X^{*} \Rightarrow T(f)=\operatorname{cl~} \int_{\Omega} f(\omega) F(\omega) d \mu(\omega)$. Q.E.D. 
Remark. If $Q \in P_{\mathrm{w} k c}(X)$, then

$$
\int_{A} F(\omega) d \mu(\omega) \subseteq \mu(A) Q=\int_{A} Q d \mu(\omega) \Rightarrow F(\omega) \subseteq Q \quad \mu \text {-a.e. }
$$

for all $A \in \Sigma$. So $F(\cdot)$ can be taken to be $P_{\mathrm{w} k c}(X)$ valued and integrably bounded and then by Theorem 4.2 of this paper $\int_{\Omega} f(\omega) F(\omega) d \mu(\omega) \in P_{\mathrm{w} k c}(X)$ for all $f(\cdot) \in L^{1}(\Omega)$ and so the representation formula in the above theorem takes the form $T(f)=\int_{\Omega} f(\omega) F(\omega) d \mu(\omega)$.

Next we are going to use Theorem 3.2 to obtain a corresponding representation result for set valued operators acting on $L^{\infty}(\Omega)$.

THEOREM 3.3. Assume that $X^{*}$ is separable too. If $T: L^{\infty}(\Omega) \rightarrow P_{f c}(X)$ is s.t. there exists $\Omega_{\varepsilon} \subseteq \Omega$ for every $\varepsilon>0$ with $\mu\left(\Omega \backslash \Omega_{\varepsilon}\right)<\varepsilon$ for which $T(f) \subseteq\|f\|_{1} Q_{\varepsilon}$, where $Q_{\varepsilon} \in P_{\mathrm{w} k c}(X)$, and whenever supp $f \subseteq \Omega_{\varepsilon}$ and $f \rightarrow \sigma_{T(f)}\left(x^{*}\right)$ is continuous on $L^{\infty}\left(\Omega_{\varepsilon}\right)$ for all $x^{*} \in X^{*}$ and all $\varepsilon>0$, and for all $f_{n} \stackrel{\mathrm{w}-L^{1}}{\rightarrow} f$ we have $\mathrm{w}-\underline{\lim }_{n \rightarrow \infty} T\left(f_{n}\right)=T(f)$, then there exists $F: \Omega \rightarrow P_{\mathrm{w} k c}(X)$ scalarly integrable s.t.

$$
T(f)=\operatorname{cl} \int_{\Omega} f(\omega) F(\omega) d \mu(\omega) \quad \text { for all } f \in L^{\infty}(\Omega) .
$$

Proof. Let $\left\{\Omega_{n}\right\}_{n \geqslant 1} \subseteq \Sigma$ with $\mu\left(\Omega_{n}\right) \uparrow \mu(\Omega)$ and $\left\{Q_{n}\right\}_{n \geqslant 1} \subseteq H\left(X, X^{*}\right)$ be sequences of sets in $\Omega$ and $X$ respectively, postulated by the hypotheses of the theorem. Then from Theorem 3.2 we know that for all $n \geqslant 1$ there exist $F_{n}: \Omega \rightarrow P_{\mathrm{w} k c}(X)$ scalarly integrable s.t. $T(f)=\operatorname{cl} \int_{\Omega} f(\omega) F_{n}(\omega) d \mu(\omega)$ for all $f(\cdot) \in L^{\infty}\left(\Omega_{n}\right)$. Suppose $\mu\left(\Omega_{n} \cap \Omega_{m}\right)>0$. For all $B \in\left[\Sigma \cap\left(\Omega_{n} \cap \Omega_{m}\right)\right]$ we have that cl $\int_{B} F_{n}(\omega) d \mu(\omega)$ $=\operatorname{cl} \int_{B} F_{m}(\omega) d \mu(\omega)=T\left(\chi_{B}\right)$. Then Lemma 4.2 of Hiai and Umegaki [18] tells us that

$$
S_{F_{n} \backslash \Omega_{n} \cap \Omega_{m}}^{1}=S_{F_{n} \mid \Omega_{n} \cap \Omega_{m}}^{1} \Rightarrow F_{n}(\omega)=F_{m}(\omega) \quad \mu_{\Omega_{n} \cap \Omega_{m}} \text {-a.e. }
$$

To take care of those exceptional $\mu$-null sets and of the case where $\mu\left(\Omega_{n} \cap \Omega_{m}\right)=0$ and $F_{n}(\omega) \neq F_{m}(\omega)$ there, call those sets $N_{n m}$ and let $N_{n}=\bigcup_{m=1}^{\infty} N_{n m}$ and $N^{\prime}=$ $\cup_{n=1}^{\infty} N_{n}$. Clearly $\mu\left(N^{\prime}\right)=0$. Also let $N^{\prime \prime}=\Omega \backslash \bigcup_{n=1}^{\infty} \Omega_{n}$. Again $\mu\left(N^{\prime \prime}\right)=0$. Set $N=N^{\prime} \cup N^{\prime \prime}$ and define

$$
F(\omega)= \begin{cases}F_{n}(\omega) & \text { for } \omega \in \Omega_{n} \backslash N_{n} \\ \{0\} & \text { for } \omega \in N\end{cases}
$$

What preceded the definition of $F(\cdot)$ shows that this is a well-defined multifunction. Our claim is that for all $f \in L^{\infty}(\Omega)$

$$
T(f)=\operatorname{cl} \int_{\Omega} f(\omega) F(\omega) d \mu(\omega) .
$$

Let $g \in S_{F}^{1}$ and consider $\left\{g_{n}=\chi_{\Omega_{n}} g\right\}_{n \geqslant 1}$ and $\left\{f_{n}=\chi_{\Omega_{n}} f\right\}_{n \geqslant 1}$. Then $f_{n} \cdot g_{n} \in$ $L_{X}^{1}(\Omega)$ and $T\left(f_{n}\right)=\int_{\Omega} f_{n}(\omega) g_{n}(\omega) d \mu(\omega)$. Since $f_{n} \cdot g_{n} \stackrel{L_{x}^{1}}{\rightarrow} f \cdot g$ we have that $\int_{\Omega} f_{n}(\omega) g_{n}(\omega) d \mu(\omega) \stackrel{\mathrm{s}}{\rightarrow} \int_{\Omega} f(\omega) g(\omega) d \mu(\omega)$. Also recall that $\mathrm{w}-\underline{\lim }_{n \rightarrow \infty} T\left(f_{n}\right)=$ $T(f)$. Hence

$$
\int_{\Omega} f(\omega) g(\omega) d \mu(\omega) \in T(f) \Rightarrow \operatorname{cl} \int_{\Omega} f(\omega) F(\omega) d \mu(\omega) \subseteq T(f) .
$$


Suppose that in fact strict inclusion holds. So thete exists $u \in T(f)$ s.t. $u \notin$ cl $\int_{\Omega} f(\omega) F(\omega) d \mu(\omega)$. Then from the strong separation theorem we know that there exist $\varepsilon>0$ and nonzero $x^{*} \in X^{*}$ s.t.

$$
\sigma_{f_{\Omega} f F}\left(x^{*}\right)=\int_{\Omega} \sigma_{f(\omega) F(\omega)}\left(x^{*}\right) d \mu(\omega) \leqslant\left(x^{*}, u\right)-\varepsilon .
$$

Apply Aumann's selection theorem to find $g \in S_{F}^{1}$ s.t.

$$
\begin{aligned}
\int_{\Omega} \sigma_{f(\omega) F(\omega)}\left(x^{*}\right) d \mu(\omega) & =\int_{\Omega}\left(x^{*}, f(\omega) g(\omega)\right) d \mu(\omega) \\
& =\left(x^{*}, \int_{\Omega} f(\omega) g(\omega) d \mu(\omega)\right) .
\end{aligned}
$$

Thus we have that $\left(x^{*}, \int_{\Omega} f(\omega) g(\omega) d \mu(\omega)\right) \leqslant\left(x^{*}, u\right)-\varepsilon$. Recall that $\int_{\Omega} f_{n}(\omega) g_{n}(\omega) d \mu(\omega) \stackrel{s}{\rightarrow} \int_{\Omega} f(\omega) g(\omega) d \mu(\omega)$. So for $n \geqslant n_{1}$ we have

$$
\left(x^{*}, \int_{\Omega} f_{n}(\omega) g_{n}(\omega) d \mu(\omega)\right) \leqslant\left(x^{*}, \int_{\Omega} f(\omega) g(\omega) d \mu(\omega)\right)+\varepsilon / 3 .
$$

Once again we recall that $\mathrm{w}-\lim _{n} T\left(f_{n}\right)=T(f)$. Hence we can find $u_{n} \in T\left(f_{n}\right)$ s.t. $u_{n} \stackrel{\mathrm{w}}{\rightarrow} u$. Then for $n \geqslant n_{2}$ we will have

$$
\left(x^{*}, u\right)-\varepsilon / 3 \leqslant\left(x^{*}, u_{n}\right) .
$$

Combining (1) and (2) above we deduce that for $n \geqslant \max \left(n_{1}, n_{2}\right)$

$$
\left(x^{*}, \int_{\Omega} f_{n}(\omega) g_{n}(\omega) d \mu(\omega)\right) \leqslant\left(x^{*}, u_{n}\right)-\varepsilon / 3 .
$$

Note that $g_{n} \in S_{F_{n}}^{1}$. We claim that $\left(x^{*}, f_{n}(\omega) g_{n}(\omega)\right)=\sigma_{f_{n}(\omega) F_{n}(\omega)}\left(x^{*}\right) \mu_{\Omega_{n}}$-a.e. Suppose not. Then for some $A \in \Sigma \cap \Omega_{n}$ with $\mu_{\Omega_{n}}(A)=\mu(A)>0$ we have that

$$
\begin{aligned}
\left(x^{*}, f_{n}(\omega) g_{n}(\omega)\right) & <\sigma_{f_{n}(\omega) F_{n}(\omega)}\left(x^{*}\right) \text { for } \omega \in A \\
& \Rightarrow \int_{\Omega_{n}}\left(x^{*}, f_{n}(\omega) g_{n}(\omega)\right) d \mu(\omega)<\int_{\Omega_{n}} \sigma_{f_{n}(\omega) F_{n}(\omega)}\left(x^{*}\right) d \mu(\omega) .
\end{aligned}
$$

But $\sigma_{f(\omega) F(\omega)}\left(x^{*}\right)=\left(x^{*}, f(\omega) g(\omega)\right) \mu$-a.e. So

$$
\begin{aligned}
\int_{\Omega_{n}} \sigma_{f(\omega) F(\omega)}\left(x^{*}\right) d \mu(\omega) & =\left(x^{*}, \int_{\Omega_{n}} f(\omega) g(\omega) d \mu(\omega)\right) \\
& \Rightarrow \int_{\Omega_{n}} \sigma_{f_{n}(\omega) F_{n}(\omega)}\left(x^{*}\right) d \mu(\omega) \\
& =\left(x^{*}, \int_{\Omega_{n}} f_{n}(\omega) g_{n}(\omega) d \mu(\omega)\right) .
\end{aligned}
$$

From (4) and (5) we get a contradiction. Hence indeed

$$
\sigma_{f_{n}(\omega) F_{n}(\omega)}\left(x^{*}\right)=\left(x^{*}, f_{n}(\omega) g_{n}(\omega)\right) \quad \mu_{\Omega_{n}} \text {-a.e. }
$$


Using that in (3) we get

$$
\begin{aligned}
& \int_{\Omega_{n}} \sigma_{f_{n}(\omega) F_{n}(\omega)}\left(x^{*}\right) d \mu(\omega) \leqslant\left(x^{*}, u_{n}\right)-\varepsilon / 3 \\
& \Rightarrow \sigma_{\Omega_{\Omega_{n}} f_{n} F_{n}}\left(x^{*}\right) \leqslant\left(x^{*}, u_{n}\right)-\varepsilon / 3 \\
& \Rightarrow u_{n} \notin T\left(f_{n}\right)=\int_{\Omega_{n}} f_{n}(\omega) F_{n}(\omega) d \mu(\omega)
\end{aligned}
$$

for $n \geqslant \max \left(n_{1}, n_{2}\right)$, which is a contradiction. So finally we conclude that $T(f)=$ $\int_{\Omega} f(\omega) F(\omega) d \mu(\omega)$. Q.E.D.

We will close this section with another representation theorem for set valued operators on $L^{\infty}(\Omega)$ which are continuous with respect to the Kuratowski-Mosco convergence of sets, when the arguments in $L^{\infty}(\Omega)$ converge strongly.

TheOREM 3.4. Assume that $(\Omega, \Sigma, \mu)$ is nonatomic and $X$ is a separable reflexive Banach space. If $T: L^{\infty} \rightarrow P_{f c}(X)$ is linear, $f \rightarrow T(f)$ is continuous on $L^{\infty}(\Omega)$ for the $K-M$ convergence and $T\left(\chi_{A}\right) \subseteq \mu(A) Q$ for all $A \in \Sigma$, where $Q$ is closed and bounded, then there exists $F: \Omega \rightarrow P_{f c}(X)$ integrably bounded s.t. $T(f)=\int_{\Omega} f(\omega) F(\omega) d \mu(\omega)$ for all $f \in L^{\infty}(\Omega)$.

Proof. As before we can have that $A \rightarrow \sigma_{T\left(\chi_{A}\right)}\left(x^{*}\right)$ is a signed measure for all $x^{*} \in X^{*}$. Since $T(\cdot)$ has values in $P_{\mathrm{w} k c}(X)$, from Theorem 1.1 of Costé [7] we deduce that $M: A \rightarrow T\left(\chi_{A}\right)$ is a strong multimeasure in the sense of that paper.

Also from our boundedness assumption we can see that $M(\cdot)$ is $\mu$-continuous and of bounded variation. Invoking Theorem 4.5 of Hiai [16] we get that $M(\cdot)$ has a set valued Radon-Nikodým derivative $F: \Omega \rightarrow P_{f}(X)$ which is integrably bounded. Because $(\Omega, \Sigma, \mu)$ is nonatomic we can take $F(\cdot)$ to be convex valued. Also note that $F(\omega) \subseteq Q \mu$-a.e. So we have

$$
T\left(\chi_{A}\right)=\int_{A} F(\omega) d \mu(\omega)
$$

with $F: \Omega \rightarrow P_{\text {wkc }}(X)$ is integrably bounded.

Let

$$
\begin{aligned}
s(\cdot) & =\sum_{k=1}^{N} \chi_{A_{k}}(\cdot) r_{k} \Rightarrow T(s)=\sum_{k=1}^{N} T\left(\chi_{A_{k}} r_{k}\right)=\sum_{k=1}^{N} r_{k} T\left(\chi_{A_{k}}\right) \\
& =\sum_{k=1}^{N} r_{k} \int_{A_{k}} F(\omega) d \mu(\omega)=\sum_{k=1}^{N} \int_{\Omega} \chi_{A_{k}}(\omega) r_{k} F(\omega) d \mu(\omega) \\
& =\int_{\Omega} \sum_{k=1}^{N} \chi_{A_{k}}(\omega) r_{k} F(\omega) d \mu(\omega)=\int_{\Omega} s(\omega) F(\omega) d \mu(\omega) .
\end{aligned}
$$

Next let $\left\{s_{n}\right\}_{n \geqslant 1}$ be simple functions s.t. $s_{n} \stackrel{L^{\infty}}{\rightarrow} f$ (see Hewitt and Stromberg [15, Theorem 11.35 , p. 159]). We claim that

$$
\int_{\Omega} s_{n}(\omega) F(\omega) d \mu(\omega) \stackrel{\mathrm{K}-\mathrm{M}}{\rightarrow} \int_{\Omega} f(\omega) F(\omega) d \mu(\omega) .
$$


So let $x \in \mathrm{w}-\varlimsup \lim _{\Omega} s_{n}(\omega) F(\omega) d \mu(\omega)$. Then $x=\mathrm{w}-\lim _{k \rightarrow \infty} x_{n_{k}}$ with $x_{n_{k}} \in$ $\int_{\Omega} s_{n_{k}}(\omega) F(\omega) d \mu(\omega)$. Let $x_{n_{k}}=\int_{\Omega} s_{n_{k}}(\omega) g_{n_{k}}(\omega) d \mu(\omega)$, where $g_{n_{k}}(\cdot) \in S_{F}^{1}$. Theorem 4.2 of this paper (see also Proposition 3.1 of [24]) tells us that $S_{F}^{1}$ is w-compact in $L_{X}^{1}(\Omega)$. So from the Eberlein-Smulian theorem we deduce that there is a subsequence of $\left\{g_{n_{k}}(\cdot)\right\}_{k \geqslant 1}$ (which for simplicity we denote with the same index) s.t.

$$
g_{n_{k}} \stackrel{\text { w- } L_{x}^{1}}{\rightarrow} g \in S_{F}^{1} .
$$

Then for every $x^{*} \in X^{*}$ we have that

$$
\begin{aligned}
\mid\left(x^{*}, \int_{\Omega}\right. & \left.s_{n_{k}}(\omega) g_{n_{k}}(\omega) d \mu(\omega)\right)-\left(x^{*}, \int_{\Omega} f(\omega) g(\omega) d \mu(\omega)\right) \mid \\
= & \mid\left(x^{*}, \int_{\Omega} s_{n_{k}}(\omega) g_{n_{k}}(\omega) d \mu(\omega)\right)-\left(x^{*}, \int_{\Omega} f(\omega) g_{n_{k}}(\omega) d \mu(\omega)\right) \\
& +\left(x^{*}, \int_{\Omega} f(\omega) g_{n_{k}}(\omega) d \mu(\omega)\right)-\left(x^{*}, \int_{\Omega} f(\omega) g(\omega) d \mu(\omega)\right) \mid \\
\leqslant & \left|\left(x^{*}, \int_{\Omega}\left(s_{n_{k}}(\omega)-f(\omega)\right) g_{n_{k}}(\omega) d \mu(\omega)\right)\right| \\
& +\left|\left(x^{*}, \int_{\Omega} f(\omega)\left(g_{n_{k}}(\omega)-g(\omega)\right) d \mu(\omega)\right)\right| \\
= & \left|\int_{\Omega}\left(s_{n_{k}}(\omega)-f(\omega)\right)\left(x^{*}, g_{n_{k}}(\omega)\right) d \mu(\omega)\right| \\
& +\left|\int_{\Omega} f(\omega)\left(x^{*}, g_{n_{k}}(\omega)-g(\omega)\right) d \mu(\omega)\right| \\
= & \left|\left\langle\left(s_{n_{k}}-f\right) x^{*}, g_{n_{k}}\right\rangle\right|+\left|\left\langle f x^{*}, g_{n_{k}}-g\right\rangle\right|,
\end{aligned}
$$

where $\langle\cdot, \cdot\rangle$ denotes the duality brackets between $L_{X^{*}}^{\infty}(\Omega)$ and $L_{X}^{1}(\Omega)$. Note that

$$
s_{n_{k}} x^{*} \stackrel{s-L_{X^{*}}^{\infty}}{\rightarrow} f x^{*} \text { and } g_{n_{k}} \stackrel{\text { w- } L_{X}^{1}}{\rightarrow} g \quad \text { as } k \rightarrow \infty \text {. }
$$

So passing to the limit we get that

$$
\begin{aligned}
\left(x^{*}, \int_{\Omega} s_{n_{k}}(\omega) g_{n_{k}}(\omega) d \mu(\omega)\right) & \rightarrow\left(x^{*}, \int_{\Omega} f(\omega) g(\omega) d \mu(\omega)\right) \\
& \Rightarrow x_{n_{k}}=\int_{\Omega} s_{n_{k}}(\omega) g_{n_{k}}(\omega) d \mu(\omega) \\
& \stackrel{\mathrm{w}}{\rightarrow} \int_{\Omega} f(\omega) g(\omega) d \mu(\omega) \in \int_{\Omega} f(\omega) F(\omega) d \mu(\omega) .
\end{aligned}
$$

On the other hand, we also have that $x_{n_{k}} \stackrel{\mathrm{w}}{\rightarrow} x$. So $x=\int_{\Omega} f(\omega) g(\omega) d \mu(\omega)$ which implies that

$$
\underset{n \rightarrow \infty}{\mathrm{w}-\varlimsup_{\Omega}} \int_{\Omega} s_{n}(\omega) F(\omega) d \mu(\omega) \subseteq \int_{\Omega} f(\omega) F(\omega) d \mu(\omega) .
$$


Next let $x \in \int_{\Omega} f(\omega) F(\omega) d \mu(\omega)$. Then there exists $g(\cdot) \in S_{F}^{1}$ s.t. $x=$ $\int_{\Omega} f(\omega) g(\omega) d \mu(\omega)$. Let

$$
x_{n}=\int_{\Omega} s_{n}(\omega) g(\omega) d \mu(\omega) \in \int_{\Omega} s_{n}(\omega) F(\omega) d \mu(\omega)
$$

We have

$$
\left\|x_{n}-x\right\| \leqslant \int_{\Omega}\left|s_{n}(\omega)-f(\omega)\right|\|g(\omega)\| \rightarrow 0 \quad \text { as } n \rightarrow \infty .
$$

So $x \in \mathrm{s}-\underline{\lim }_{n \rightarrow \infty} \int_{\Omega} s_{n}(\omega) F(\omega) d \mu(\omega)$ which means that

$$
\int_{\Omega} f(\omega) F(\omega) d \mu(\omega) \subseteq \mathrm{s}-\underset{n \rightarrow \infty}{\lim } \int_{\Omega} s_{n}(\omega) F(\omega) d \mu(\omega) .
$$

From (6) and (7) we get that

$$
\begin{aligned}
\int_{\Omega} s_{n}(\omega) F(\omega) d \mu(\omega) & \stackrel{\mathrm{K}-\mathrm{M}}{\rightarrow} \int_{\Omega} f(\omega) F(\omega) d \mu(\omega) \\
& \Rightarrow T\left(s_{n}\right) \stackrel{\mathrm{K}-\mathrm{M}}{\rightarrow} \int_{\Omega} f(\omega) F(\omega) d \mu(\omega) .
\end{aligned}
$$

On the other hand, since $T(\cdot)$ is continuous for the K-M convergence on $L^{\infty}(\Omega)$ we have that $T\left(s_{n}\right) \stackrel{\mathrm{K}-\mathrm{M}}{\rightarrow} T(f)$. Recall that the Kuratowski-Mosco limit is unique. So $T(f)=\int_{\Omega} f(\omega) F(\omega) d \mu(\omega)$. Q.E.D.

4. Integrable multifunctions. In this section we will examine some properties of the representation integrals obtained in the previous section and then we will prove a differentiability kind of result for a large class of multifunctions and we will determine a necessary and sufficient condition for $S_{F}^{1}$ to be w-compact in $L_{X}^{1}$, generalizing an earlier result by Diestel [11].

In the first result we characterize the extremal points of a representable set valued operator $T: L^{1} \rightarrow P_{f c}(X)$.

Proposition 4.1. If $T(f)=\int_{\Omega} f(\omega) F(\omega) d \mu(\omega)$, where $F: \Omega \rightarrow P_{\mathrm{w} k c}(X)$ is integrably bounded, then ext $T(f) \subseteq \int_{\Omega} f(\omega) \operatorname{ext} F(\omega) d \mu(\omega)$.

Proof. Let $x \in \operatorname{ext} T(f)$. Then $x=\int_{\Omega} f(\omega) g(\omega) d \mu(\omega)$ for $g(\cdot) \in S_{F}^{1}$. Since we know that $S_{\text {ext } F}^{1}=$ ext $S_{F}^{1}$ from Castaing and Valadier [6], if $g \notin S_{\text {ext } F}^{1}$ then there must be $g_{1}, g_{2} \in S_{F}^{1}$ s.t. $g=\frac{1}{2} g_{1}+\frac{1}{2} g_{2}$. Hence

$$
\begin{aligned}
x & =\int_{\Omega} f(\omega) g(\omega) d \mu(\omega)=\int_{\Omega} f(\omega)\left[\frac{1}{2} g_{1}(\omega)+\frac{1}{2} g_{2}(\omega)\right] d \mu(\omega) \\
& =\frac{1}{2} \int_{\Omega} f(\omega) g_{1}(\omega) d \mu(\omega)+\frac{1}{2} \int_{\Omega} f(\omega) g_{2}(\omega) d \mu(\omega)=\frac{x_{1}+x_{2}}{2}
\end{aligned}
$$

with $x_{1}, x_{2} \in T(f)=\int_{\Omega} f(\omega) F(\omega) d \mu(\omega)$. But this then contradicts the extremality of $x$. Q.E.D.

We have another characterization of the exposed points of a set valued operator $T(\cdot)$ acting on $L^{1}(\Omega)$.

Assume that $X$ has the R.N.P. 
Proposition 4.2. If $T: L^{1} \rightarrow P_{f c}(X)$ is additive, $f \rightarrow \sigma_{T(f)}\left(x^{*}\right)$ is continuous for all $x^{*} \in X^{*}, T\left(f_{0}\right) \in P_{\mathrm{w} k c}(X)$ for $f_{0} \in L^{1}(\Omega)$ and $\left|T\left(\chi_{B} f_{0}\right)\right| \leqslant \mu(B)\left\|f_{0}\right\|_{1}$, where $B \in \Sigma$, and $x \in \exp T\left(f_{0}\right)$, then there exists $g_{f_{0}}(\cdot) \in L_{X}^{1}(\Omega)$ s.t. $x=\int_{\Omega} g_{f_{0}}(\omega) d \mu(\omega)$.

REMARK. Note that such a set valued operator is not necessarily representable and so we cannot get the proposition as a consequence of one of the representation theorems of $\S 3$.

Proof. As before we can show that $A \rightarrow \sigma_{T\left(\chi_{A} f_{0}\right)}\left(x^{*}\right)$ for all $x^{*} \in X^{*}$ is a signed measure. Since $T\left(f_{0}\right) \in P_{\mathrm{w} k c}(X)$, Theorem 1.1 of Costé [7] tells us that $M$ : $A \rightarrow T\left(\chi_{A} f_{0}\right)$ is a strong multimeasure which is clearly $\mu$-continuous and of bounded variation. So we can use Proposition 2.1 of Hiai [16] and get a vector measure $m_{f_{0}}$ : $\Sigma \rightarrow X$ s.t. $m_{f_{0}}(\Omega)=x$. Note that $m_{f_{0}}(\cdot)$ being a measure selector of $M(\cdot)$ is $\mu$-continuous and of bounded variation. Since $X$ has the R.N.P. we deduce that there exists $g_{f_{0}}(\cdot) \in L_{X}^{1}(\Omega)$ s.t. $x=\int_{\Omega} g_{f_{0}}(\omega) d \mu(\omega)$. Q.E.D.

Now we will have the differentiation result for multifunctions which extends the single valued result of Gelfand (see [21]) and the earlier multivalued results of Artstein [2], Castaing [5] and Hermes [14]. Assume $X$ is reflexive.

THEOREM 4.1. If $\Phi:[0, T] \rightarrow P_{f c}(X)$ is measurable and for all $x^{*} \in X^{*}$ and all $t_{0}$, $t_{1} \in[0, T]$ we have that

$$
\left|\sigma_{\Phi\left(t_{0}\right)}\left(x^{*}\right)-\sigma_{\Phi\left(t_{1}\right)}\left(x^{*}\right)\right| \leqslant\left\|x^{*}\right\|\left|t_{1}-t_{0}\right|,
$$

then there exists $F: \Omega \rightarrow P_{f c}(X)$ scalarly integrable s.t. $\Phi\left(t_{1}\right)=\Phi\left(t_{0}\right)+\operatorname{cl} \int_{t_{0}}^{t_{1}} F(t) d t$ for all $t_{0}, t_{1} \in[0, T]$.

Proof. From Dinculeanu [13] we know that to $\sigma_{\Phi(t)}\left(x^{*}\right)$ corresponds a measure $m_{x^{*}}(\cdot)$ s.t.

$$
m_{x^{*}}\left[t_{0}, t_{1}\right]=\sigma_{\Phi\left(t_{1}\right)}\left(x^{*}\right)-\sigma_{\Phi\left(t_{0}\right)}\left(x^{*}\right)
$$

and

(*) $\quad\left|m_{x^{*}}\right|(A) \leqslant\left\|x^{*}\right\| \lambda(A), \quad A \in \Sigma=$ Lebesgue sets of $[0, T]$,

where $\lambda(\cdot)$ is the Lebesgue measure on $[0, T]$. From the Radon-Nikodým Theorem we know that there exists $\varphi\left(x^{*}, \cdot\right) \in L^{1}$ s.t.

$$
m_{x^{*}}(A)=\int_{A} \varphi\left(x^{*}, t\right) d t
$$

for every Lebesgue measurable set $A$ in $[0, T]$. We claim that $\varphi\left(x^{*}, \cdot\right) \in L^{\infty}$. Suppose not. Then for every $n \geqslant 1$ there exist $A_{n} \in \Sigma=$ Lebesgue measurable sets in $[0,1]$ with $\mu\left(A_{n}\right)>0$ s.t. $\left|\varphi\left(x^{*}, t\right)\right|>n$ for $t \in A_{n}$. Hence we have that

$$
\begin{aligned}
\left|m_{x^{*}}\right|\left(A_{n}\right) & =\int_{A_{n}}\left|\varphi\left(x^{*}, t\right)\right| d t>n \cdot \lambda\left(A_{n}\right) \\
& \Rightarrow \frac{\left|m_{x^{*}}\right|\left(A_{n}\right)}{\lambda\left(A_{n}\right)}>n
\end{aligned}
$$

which shows that $\left\{\left|m_{x^{*}}\right|(A) / \lambda(A): A \in \Sigma, \lambda(A)>0\right\}$ is unbounded, contradicting (*). Thus $\varphi\left(x^{*}, \cdot\right) \in L^{\infty}$. From $(* *)$ we see that $x^{*} \rightarrow \varphi\left(x^{*}, t\right)$ is sublinear for $t \in[0, T] \backslash N_{x^{*}}$, where $\lambda\left(N_{x^{*}}\right)=0$. By a lifting argument as in the proof of Theorem 
3.1 we can get that $x^{*} \rightarrow \hat{\varphi}\left(x^{*}, t\right)=\rho\left[\varphi\left(x^{*}, t\right)\right]$ is sublinear for all $t \in[0, T]$. Also it is continuous. So applying Hörmander's theorem we can find $F: \Omega \rightarrow P_{f c}(X)$ s.t. $\hat{\varphi}\left(x^{*}, t\right)=\sigma_{F(t)}\left(x^{*}\right)$. So $F(\cdot)$ is scalarly integrable (and so $F(\cdot)$ is measurable by Theorem III-37 of Castaing and Valadier [6]). Hence we have

$$
\begin{aligned}
m_{x^{*}}(A) & =\int_{A} \sigma_{F(t)}\left(x^{*}\right) d t \\
& \Rightarrow \sigma_{\Phi\left(t_{1}\right)}\left(x^{*}\right)=\sigma_{\Phi\left(t_{0}\right)}\left(x^{*}\right)+\int_{t_{0}}^{t_{1}} \sigma_{F(t)}\left(x^{*}\right) d t \\
& \Rightarrow \sigma_{\Phi\left(t_{1}\right)}\left(x^{*}\right)=\sigma_{\Phi\left(t_{0}\right)}\left(x^{*}\right)+\sigma_{f_{t_{0}} t_{(t)}(t) d t}\left(x^{*}\right) .
\end{aligned}
$$

Since this is true for all $x^{*} \in X^{*}$ we conclude that

$$
\Phi\left(t_{1}\right)=\Phi\left(t_{0}\right)+\mathrm{cl} \int_{t_{0}}^{t_{1}} F(t) d t \text { Q.E.D. }
$$

We will close the paper with a result that we have already used in several places in this work. This is a necessary and sufficient condition for the set of integrable selectors of a multifunction to be w-compact in $L_{X}^{1}(\Omega)$. The sufficiency part was first obtained by the author in [24]. The necessity part is new. Furthermore the sufficiency part generalizes the result of Diestel [11] and provides a different approach to the problem; namely instead of the Davis-Figiel-Johnson-Pelczynski factorization method (see [11]) we use techniques from the general theory of multifunctions and the celebrated James theorem for weak compactness.

Assume that $(\Omega, \Sigma, \mu)$ is nonatomic and $X^{*}$ is separable.

THEOREM 4.2. If $F: \Omega \rightarrow P_{f}(X)$ is integrably bounded, then $S_{F}^{1}$ is w-compact in $L_{X}^{1}(\Omega)$ if and only if $F(\cdot)$ has values in $P_{\mathrm{w} k c}(X) \mu$-a.e.

Proof. Assume $S_{F}^{1}$ is w-compact in $L_{X}^{1}(\Omega)$. Because $(\Omega, \Sigma, \mu)$ is nonatomic from Cuong [9] we know that $S_{F}^{1}$ is weakly dense in $\overline{\operatorname{conv}} S_{F}^{1}=S_{\text {conv } F}^{1}$. Since $S_{F}^{1}$ is w-closed we deduce that $S_{F}^{1}=S \frac{1}{\operatorname{conv} F} \Rightarrow F(\omega)=\overline{\operatorname{conv}} F(\omega) \mu$-a.e. So $F(\cdot)$ is $\mu$-a.e. convex valued.

Next let $x^{*} \in X^{*}$. Then from James' theorem for some $\hat{f} \in S_{F}^{1}$ we have that

$$
\sigma_{S_{F}^{1}}\left(x^{*}\right)=\left\langle x^{*}, \hat{f}\right\rangle=\left(x^{*}, \int_{\Omega} \hat{f}(\omega) d \mu(\omega)\right) .
$$

Also from Theorem 2.2 of Hiai and Umegaki [18] we can easily see that

$$
\sigma_{S_{F}^{1}}\left(x^{*}\right)=\int_{\Omega} \sigma_{F(\omega)}\left(x^{*}\right) d \mu(\omega) .
$$

So we have

$$
\int_{\Omega}\left[\sigma_{F(\omega)}\left(x^{*}\right)-\left(x^{*}, \hat{f}(\omega)\right)\right] d \mu(\omega)=0 .
$$

But note that since $\hat{f}(\cdot) \in S_{F}^{1}$ and $F(\cdot)$ is $\mu$-a.e. convex valued we have that $\left(x^{*}, \hat{f}(\omega)\right) \leqslant \sigma_{F(\omega)}\left(x^{*}\right) \mu$-a.e. Hence,

$$
\sigma_{F(\omega)}\left(x^{*}\right)=\left(x^{*}, \hat{f}(\omega)\right)
$$


for all $\omega \in \Omega \backslash N_{x^{*}}$, where $\mu\left(N_{x^{*}}\right)=0$. Since $X^{*}$ is separable and $\sigma_{F(\omega)}(\cdot)$ is continuous we can easily see that

$$
\sigma_{F(\omega)}\left(x^{*}\right)=\left(x^{*}, \hat{f}(\omega)\right) \quad \mu \text {-a.e. }
$$

with the exceptional $\mu$-null set being independent of $x^{*} \in X^{*}$. So invoking James' theorem we conclude that $F(\omega) \in P_{\mathrm{w} k c}(X) \mu$-a.e.

Now assume that $F(\omega) \in P_{\mathrm{w} k c}(X) \mu$-a.e.

By setting $F(\omega)=\{0\}$ on the exceptional $\mu$-null set we can assume without any loss of generality that $F(\omega) \in P_{w k c}(X)$ for all $\omega \in \Omega$. From the Dinculeanu-Foiaş theorem (see Dinculeanu [13] or Ionescu-Tulcea [20]) we know that $\left[L_{X}^{1}(\Omega)\right]^{*}=$ $L_{X_{w}^{*}}^{\infty}(\Omega)$. Letting $g \in L_{X_{w}^{*}}^{\infty}(\Omega)$ we have

$$
\sigma_{S_{F}^{1}}(g)=\sup _{f \in S_{F}^{1}}\langle g, f\rangle=\sup _{f \in S_{F}^{1}} \int_{\Omega}(g(\omega), f(\omega)) d \mu(\omega) .
$$

Using Theorem 2.2 of Hiai and Umegaki [18] we get that

$$
\sup _{f \in S_{F}^{1}} \int_{\Omega}(g(\omega), f(\omega)) d \mu(\omega)=\int_{\Omega} \sup _{x \in F(\omega)}(g(\omega), x) d \mu(\omega) .
$$

Let

$$
R(\omega)=\left\{\hat{x} \in F(\omega): \sup _{x \in F(\omega)}(g(\omega), x)=\sigma_{F(\omega)}(g(\omega))=(g(\omega), \hat{x})\right\} .
$$

Since $F(\omega) \in P_{\mathrm{w} k c}(X)$ for all $\omega \in \Omega$, we have that $R(\omega) \neq \varnothing$ for all $\omega \in \Omega$. Set $\varphi(\omega, x)=\sigma_{F(\omega)}(g(\omega))-(g(\omega), \hat{x})$. Clearly $\varphi\left(\cdot{ }_{3} \cdot\right)$ is a Carathéodory function and so is jointly measurable. Then observe that $\operatorname{Gr} R=\{(\omega, \hat{x}) \in \Omega \times X: \varphi(\omega, \hat{x})=0\}$, $\mathrm{Gr} F \in \Sigma \times B(X)$. So applying Aumann's selection theorem we can find $\hat{f}: \Omega \rightarrow X$ measurable s.t. $\hat{f}(\omega) \in R(\omega)$ for all $\omega \in \Omega$. Note that $\hat{f} \in S_{F}^{1}$. Hence we have

$$
\begin{aligned}
\int_{\Omega} \sup _{x \in F(\omega)}(g(\omega), x) d \mu(\omega) & =\int_{\Omega}(g(\omega), \hat{f}(\omega)) d \mu(\omega) \\
& \Rightarrow \sigma_{S_{F}^{1}}(g)=\langle g, \hat{f}\rangle .
\end{aligned}
$$

Since $g \in L_{X_{w}^{*}}^{\infty}(\Omega)$ was arbitrary, we conclude using James' theorem that $S_{F}^{1}$ is w-compact in $L_{X}^{1}(\Omega)$. Q.E.D.

REMARK. The nonatomicity of $(\Omega, \Sigma, \mu)$ and the separability of $X^{*}$ are not needed in the sufficiency part.

ACKNOWLEDGEMENT. The author is grateful to the referee for correcting some misleading remarks of the original version of this paper and for other suggestions that improved the final form of this work.

\section{REFERENCES}

1. R. A. Alò and A. de Korvin, Representation of Hammerstein operators by Nemytskii measures, J. Math. Anal. Appl. 52 (1975), 490-513.

2. Z. Artstein, On the calculus of closed, set valued functions, Indiana Univ. Math. J. 24 (1975), 433-441.

3. R. Aumann, Integrals of set valued functions, J. Math. Anal. Appl. 12 (1965), 1-12.

4. C. Castaing, Le théorème de Dunford-Pettis généralisé, Séminaire P. Lelong (Analyse) année, 1969, Lecture Notes in Math., vol. 116, Springer, Berlin, 1970, pp. 133-151.

5. Un résultat de dérivation des multi-applications, Séminaire d'Analyse Convexe. University of Montpellier (1974), Exposé no. 2. 
6. C. Castaing and M. Valadier, Convex analysis and measurable multifunctions, Lecture Notes in Math., vol. 580, Springer-Verlag, Berlin, 1977.

7. A. Costé, Set valued measures, Topology and Measure Theory (D. D. R. Zinnowitz, ed.), 1974.

8. A. Costé and R. Pallu de la Barrière, Radon Nikodym theorems for set valued measures whose values are convex and closed, Ann. Soc. Math. Polon. Series I: Comm. Math. 20 (1978), 283-309.

9. P. Van Cuong, On the density of extremal selections for measurable multifunctions, Acta Math. Vietnam. 6 (1981), 13-28.

10. J. P. Delahaye and J. Denel, The continuities of the point-to-set maps: Definitions and equivalences, Math. Programming Stud. 10 (1979), 8-12.

11. J. Diestel, Remarks on weak compactness in $L^{1}(, X)$, Glasgow Math. J. 18 (1977), 87-91.

12. J. Diestel and J. J. Uhl, Vector measures, Math. Surveys, no. 15, Amer. Math. Soc., Providence, R. I., 1977.

13. N. Dinculeanu, Vector measures, Pergamon Press, London, 1967.

14. H. Hermes, Calculus of set valued functions and control, J. Math. Mech. 18 (1968), 47-60.

15. E. Hewitt and K. Stromberg, Real and abstract analysis, Graduate Texts in Math., no. 25 , Springer-Verlag, New York, 1966.

16. F. Hiai, Radon-Nikodym theorems for set valued measures, J. Multivariate Anal. 8 (1978), 96-118.

17. 2 (1979), 300-313. Representation of additive functionals on vector valued normed Kothe spaces, Ködai Math. J.

18. F. Hiai and H. Umegaki, Integrals, conditional expectations and martingales of multivalued functions, J. Multivariate Anal. 7 (1977), 149-182.

19. C. Himmelberg, Measurable relations, Fund. Math. 87 (1975), 53-72.

20. A. Ionescu-Tulcea and C. Ionescu-Tulcea, Topics in the theory of lifting, Springer-Verlag, Berlin, 1969.

21. I. M. Gelfand, Abstrakte Functionen und Lineare Operatoren, Math. Sb. 4 (1938), 235-286.

22. V. J. Mizel and K. Sundaresan, Representation of vector nonlinear functions, Trans. Amer. Math. Soc. 159 (1971), 111-127.

23. U. Mosco, On the continuity of the Young-Fenchel transform, J. Math. Anal. Appl. 25 (1971), $518-535$.

24. N. S. Papageorgiou, On the theory of Banach valued multifunctions, Part 1: Integration and conditional expectation, J. Multivariate Anal. 16 (1985) (in press).

25. R. T. Rockafellar, Integral functionals, normal integrands and measurable selections, Nonlinear Operators and Calculus of Variations (J. P. Gossez et al., eds.), Lecture Notes in Math., vol. 543, Springer-Verlag, Berlin, 1976.

Department of Mathematics, University of Illinois, Urbana, Illinois 61801 